\title{
Kelvin Waves Structure Analysis of a Horizontal Axis Wind Turbine Tip Vortices
}

\author{
M. M. Oueslati ${ }^{1 \dagger}$, A. W. Dahmouni ${ }^{1}$ and S. Ben Nasrallah ${ }^{2}$ \\ ${ }^{1}$ Laboratory of Wind Energy Management and Waste Energy Recovery, Research and Technologies Center \\ of Energy, Ecoparck of Borj-Cedria, BP 95 Hammam lif, 2050, Tunisia \\ ${ }^{2}$ Laboratory of Thermal and Energy Systems Studies \\ National Engineering School of Monastir, Street Ibn El Jazzar, Monastir, 5000, Tunisia
}

†Corresponding Author Email: mehdi.oueslati@crten.rnrt.tn

(Received August 8, 2019; accepted January 25, 2020)

\begin{abstract}
The optimization of the wind energy conversion is one of the most important domains which was widely interested researchers. The instabilities in the wind turbine wake are one of the sources of energy loss which strongly influenced the helical tube vortex structure and are generally difficult to be quantified using experimental facilities. This paper presents a numerical investigation on the wake downstream of a horizontal axis wind turbine (HAWT) model using the Fluent software. Results were validated using experimental measurements conducted in the CRTEn wind tunnel. The Kelvin wave's theory was, also, used to analyze the deformations acting on the tip vortices. The cartography of the velocity gradient tensor components of the first tip vortex and the different families of Kelvin wave's were studied and classified according to the azimuth wavenumber. The obtained results confirm that the tip vortices meandering correspond to the helical mode of Kelvin wave's and the stretching-compression phenomenon is the most important deformation acting on the tip vortex tubes during the development of HAWT wake.
\end{abstract}

Keywords: Horizontal axis wind turbine; Three-dimensional wake; Kelvin waves; velocity gradient tensor; Flow separation; Stretching-compression.

\section{NOMENCLATURE}

$\begin{array}{ll}C 1_{\varepsilon}, C_{2 \varepsilon}, \text { and } & C_{3 \varepsilon} \quad \text { experimental constants } \\ d & \text { diameter of the rotor } \\ \vec{F} & \text { external forces } \\ G_{k} & \text { turbulence kinetic energy due to mean } \\ & \text { velocity gradients } \\ G_{b} & \text { turbulence kinetic energy due to } \\ & \text { buoyancy } \\ g & \text { gravity } \\ I & \text { tensor unit } \\ k & \text { turbulence kinetic energy } \\ \mathrm{t} & \text { time } \\ l & \text { axial wavenumber } \\ m & \text { azimuth wavenumber } \\ p & \text { pressure } \\ Q & \text { Q- criterion } \\ R & \text { rotor Radius } \\ S & \text { source term } \\ u, v, w & \text { velocity components } \\ U_{\infty} & \text { free stream velocity } \\ \mathrm{u}_{\mathrm{t}} & \text { friction velocity } \\ u_{i} & \text { instantaneous velocity } \\ u_{i}{ }^{\prime} & \text { fluctuating velocity } \\ \vec{v} & \text { velocity vector } \\ & \end{array}$

\begin{tabular}{|c|c|}
\hline$x$ & $x$-Cartesian coordinate \\
\hline$y^{+}$ & average surface wall value \\
\hline$y_{p}$ & $\begin{array}{l}\text { distance of the first grid point from the } \\
\text { rotor }\end{array}$ \\
\hline$Y_{M}$ & $\begin{array}{l}\text { contribution of fluctuating dilation in } \\
\text { compressible turbulence to overall } \\
\text { dissipation rate }\end{array}$ \\
\hline$y$ & $y$-Cartesian coordinate \\
\hline$z$ & $z$-Cartesian coordinate \\
\hline$\alpha$ & plan angle inclination \\
\hline$\beta$ & vortex angle \\
\hline$\delta_{i j}$ & Kronecker delta \\
\hline$\Delta$ & $\Delta$-criterion \\
\hline$\Delta t$ & time step \\
\hline$\varepsilon$ & dissipation rate \\
\hline$\lambda$ & tip speed ratio \\
\hline$\lambda_{2}$ & $\lambda_{2}$-creterion \\
\hline$v$ & kinematic viscosity \\
\hline$\mu$ & molecular viscosity \\
\hline$\rho$ & density of the fluid \\
\hline$\sigma$ & turbulent Prandtl number \\
\hline
\end{tabular}




\section{INTRODUCTION}

During the last decade, grid integration of horizontal axis wind turbines for electricity production has risen quickly. The important wind potential available in many countries and the development of the technological manufacturing process leads many researchers to pay much more attention to the enhancement of wind turbine performances and wind farm layout optimization. For that, many investigations were made to reduce the wake interaction effects in a wind farms which is an important factor that strongly influences the wind turbine production, as confirmed by Grant et al. (2000) and Vermeer et al. (2003). The wind turbine wake was characterized by a complex structure with a helical shape. The tip vortices rotate as a rigid body between two fluid zones: an inner zone and an outer zone. The external forces and instabilities in these two regions interact with the vortex tubes which affects its dynamic displacement, its energy content, and its structure. Consequently, the meandering phenomenon of the tip vortices will be observed in the near wake and, also, the far wake wandering downstream the wind turbine rotor as confirmed by Larsen et al. (2007). Autor's were presented different numerical and experimental methods for modeling the wake meandering of a HAWT.

Sherry et al. (2013a) conducted an experimental study focused on the tip vortex filament stability and the interaction between the tip and root vortices in the near wake of a horizontal axis wind turbine. The intrinsic physical parameters of the tip vortices such as the circulation and the vortex core diameter were studied

Massouh and Dobrev (2014) using the phaselocked average PIV measurement techniques. Also, Noura et al. (2012) interested to the effect of the yaw angle on the performance of the wind turbine and the structure of the near wake behind the rotor. Kleusberg et al. (2019) investigated the influence of upstream sheared velocity on the wake of a HAWT and in particular the tip vortex breakdown phenomenon. By a numerical simulation way, they perturbed the tip vortices harmonically to study the effect of the instabilities on the spatial growth rate for both uniform and linear inflow velocity cases. Therefore, the complexity of the wake shape, the wandering displacement of the tip vortices and the external environmental loads are the principal sources of the vortex tube deformation.

Nevertheless, the identification process of theses deformations requires expensive experimental measurement devices and facilities to control the different physical phenomena that occur during the wind turbine operation. Due to the lack of mathematical approaches that can model a realistic vortex behavior in complex flow, researchers have used different coherent structures identification methods to understand the tip vortex structure and their wandering behavior. The most used methods that were applied for wake characterization in the literature are the $\Delta$-criterion by Chong et al. (1990), the $Q$-criterion by Hunt et al. (1988), $\lambda_{2}$-criterion by
Jeong and Hussain (1995) and the Proper Orthogonal Decomposition method (POD) applied for turbulent flow by Lumley (1967).

Tossas et al. (2013) combined the actuator line method with the Large Eddy Simulation technique to model the HAWT far wake under atmospheric conditions using OpenFOAM solver. They applied the $Q$-criterion to study the dynamic wake features in atmospheric boundary layer. They concluded that the breakdown of the wake will be promptly observed as the tip speed ratio increase. Aseyev and Cal (2016) analyzed the wake of a 4 x 3 array of a model wind turbine. They applied the $Q$-criterion, the $\Delta$-criterion, and the $\lambda_{2}$-criterion to Particle Image Velocimetry (PIV) data gathered fore and aft of the last row centerline turbine. They concluded that the $\Delta$-criterion is not suitable for the characterization of the wind turbine wake. Sarmast et al. (2014) investigated the trailing tip vortices stability downstream of the wind turbine rotor. The wake was modeled using the Large Eddy Simulation coupled with the actuator line method and then perturbed by a low-amplitude excitation source. To analyze the obtained response related to the mode's frequency and the spatial growth of tip vortices, they used two different identification methods: the Dynamic Mode Decomposition (DMD) and the Proper Orthogonal Decomposition methods (POD). Bastine et al. (2014) modeled the wake of a single wind turbine using the actuator disk model and Large Eddy Simulations. Their study focused on the effect of the turbulent atmospheric boundary layer on the wind turbine wake. The POD method was used to characterize the far wake and find the most energetic of modes necessary to reconstruct the wake. Premaratne et al. (2016) conducted experimental investigations of a HAWT within typical atmospheric boundary layer in a large-scale wind tunnel. They used PIV data with the POD method to make an energetic study of the wake and the Triple Decomposition (TD) method to explore the intensive turbulent mixing process in the wake.

In addition to the mentioned methods, other theories were developed to find out the different phenomena occurring inside a vortex tube such as the Kelvin wave's theory (Kelvin, 1867; 1880). This theory confirms that during a vortex tube displacement in a perturbed fluid flow, different types of deformations propagate as a wave along the vortex filament and characterized by its azimuth wavenumber. Each mode illustrates a physical mechanism of wave propagation and effects on the vortex tube deformation.

The method was used in a wide range of applications to characterize the vortex physics, structures, and energy exchanges such as airplane wakes (Jacquin et al. 2003), laminar and turbulent flows (Arendt et al. 1998; Mongiovi et al. 2018), vortex mass transport (Weber and Ghaffari 2014), environmental and lakes investigations (Bouffard, and Lemmin 2013) and the dynamics of tornadoes.

In this paper, we will focus on the Kelvin wave's analysis to characterize the structure and stability of 
wind turbine tip vortices. So, a three-dimensional simulation of the flow over a full HAWT rotor geometry was performed. The Fluent 6.3.26 software and the standard $k-\varepsilon$ turbulence model were used to characterize the near wake behavior of a wind turbine model and to identify the deformations that acting on the tip vortices.

\section{NUMERICAL SimUlationS}

\subsection{Horizontal Axis Wind Turbine Model Description}

The considered HAWT model (Fig. 1) was formed by three blades based on the NACA 4412 airfoil. They are characterized by a constant chord length and pitch angle to minimize the parameters that can affect the blade performances. It was previously tested and experimented in the subsonic wind tunnel of the Wind Energy Laboratory in the Research and Technology Center of Energy (CRTEn). Table 1 summarizes the geometry wind turbine model.

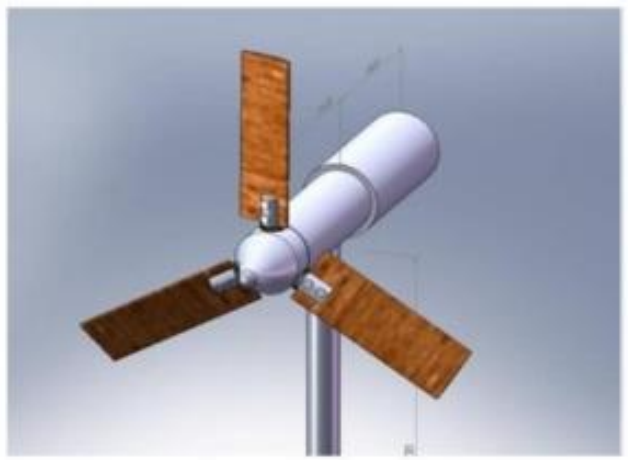

Fig. 1. Three-dimensional design of the wind turbine model.

Table 1 Geometric characteristics of the wind turbine model

\begin{tabular}{|c|c|}
\hline Parameters & Scale $(\mathrm{mm})$ \\
\hline Rotor diameter & 376 \\
\hline Hub diameter & 60 \\
\hline Nacelle length & 205 \\
\hline Length of the blade & 150 \\
\hline Constant chord length & 50 \\
\hline Fixed pitch angle & $9.5^{\circ}$ \\
\hline
\end{tabular}

\subsection{Computational Flow Field and Meshing Domain}

The three-dimensional unsteady CFD simulations of the wind turbine model were performed using Fluent software v.6.3.26 and the Gambit mesh generator. The rotor was placed in a distance of $8 \times R$ from the inlet of a rectangular duct for modeling the subsonic wind tunnel geometry. The wind tunnel have the following characteristics: a cross-section area equal to $1000 \times 800 \mathrm{~mm}^{2}$, a total length equal to $4000 \mathrm{~mm}$ corresponding to $21 \times R$ (where $R$ is the rotor radius), a maximum wind speed equal to $30 \mathrm{~m} / \mathrm{s}$ with a good stability and a turbulence intensity of $0.1 \%$. It can be operated in both cases: open or closed jet configurations.

In this study, the tower of the wind turbine was neglected to minimize the complexity of the geometry. In fact, its presence does not have a great effect on the aerodynamic performances of the rotor as mentioned by Gebhardt et al. (2010). The considered configuration is shown in Fig. 2. The computational domain was sub-divided into two sub-domains to take account of the wind turbine rotation and the wake formation. The first subdomain was a rectangular fixed zone containing the flow domain named outer region, and the second was an inner rotating region containing the rotor and the nacelle body to explore the near wake characteristics. The faces in contact with each other between the two regions ensure the continuity of the fluid flow and the relative motion of the inner zone.

The appropriate boundary conditions were set up as shown in Fig. 2. The "velocity inlet" condition was applied at the inlet of the computational domain for $8 \mathrm{~m} / \mathrm{s}$ upstream velocity, and the "pressure outlet" condition at the outlet of the channel. A "non-slip" condition was defined on the channel walls and the nacelle; however, a "moving wall" was imposed on the wind turbine rotor. In this work, the main objective is to implement a CFD model for a quick calculation of wake structure aiming to identify the types of instabilities acting on tip vortices. For that, the Moving Reference Frame (MRF) condition was activated for the fluid flow around the rotor and the near wake region to model problems involving moving parts. When this option is enabled, the equations of motion are modified to incorporate the terms of Coriolis acceleration occurring due to the transformation of a fixed reference system to a moving reference system. The MRF approach has many advantages, such as the station capacity optimization for calculations and a good compromise between results accuracy and computational time reduction.

In fact, the MRF technique models the blade loading effect by applying an axial induction factor producing a velocity deficit which is the same effect that blade loading induces on the wake by extracting kinetic energy from the free-stream wind.

Also it can model the effects of centrifugal pumping force on the fluid dynamic behavior along a wind turbine blade as mentioned by Lanzafame et al. (2013, 2016). Tran et al. 2014 and Tran and Kim (2015), studied the wake behavior of a pitching floating offshore wind turbine. They obtained the wake at different position with good accuracy during the three-dimensional CFD unsteady flow simulations using the MRF technique. The helical structure of the wake was well modeled. Consequently, the method can capture the tip and root vortices detached from the blades downstream the rotor as well as the interaction among the rotor blades and wake regimes. Besides, Moshfeghi et al. (2017) used the MRF method to highlight the flow separation features along wind turbine blades during their three-dimensional unsteady-state flow simulations. Despite that the method is a steady- 


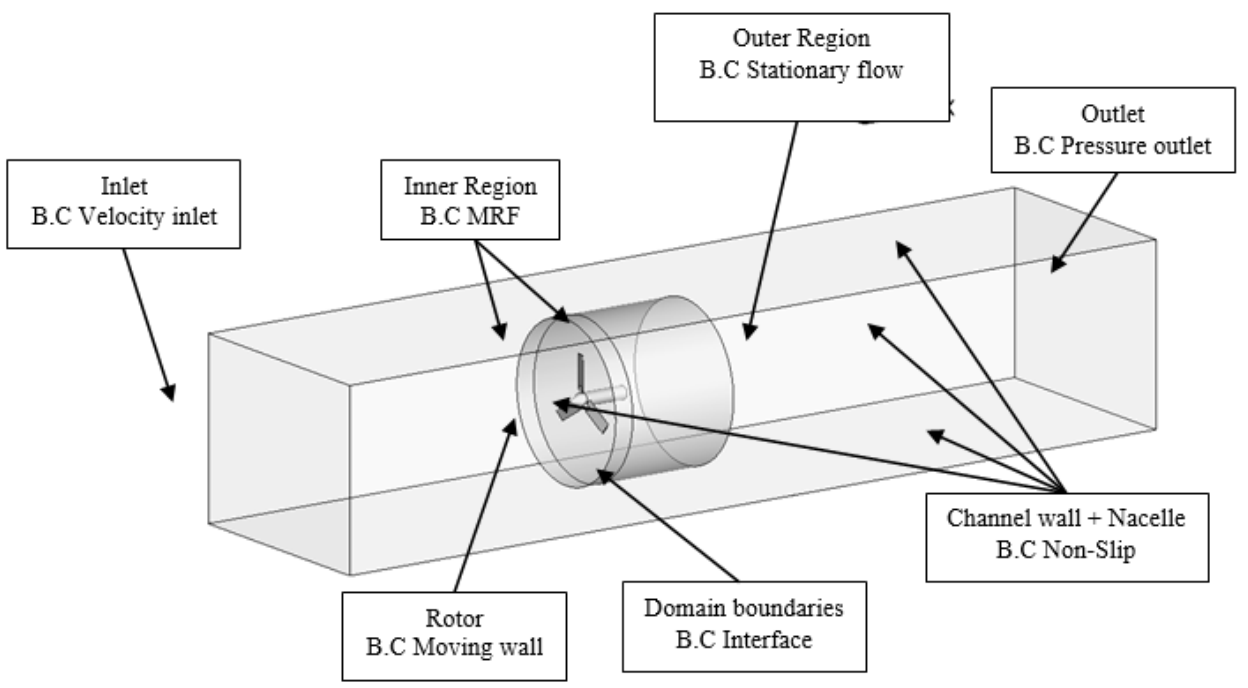

Fig. 2. Wind turbine rotor in the plane $(x, y, z)$ and boundary conditions.
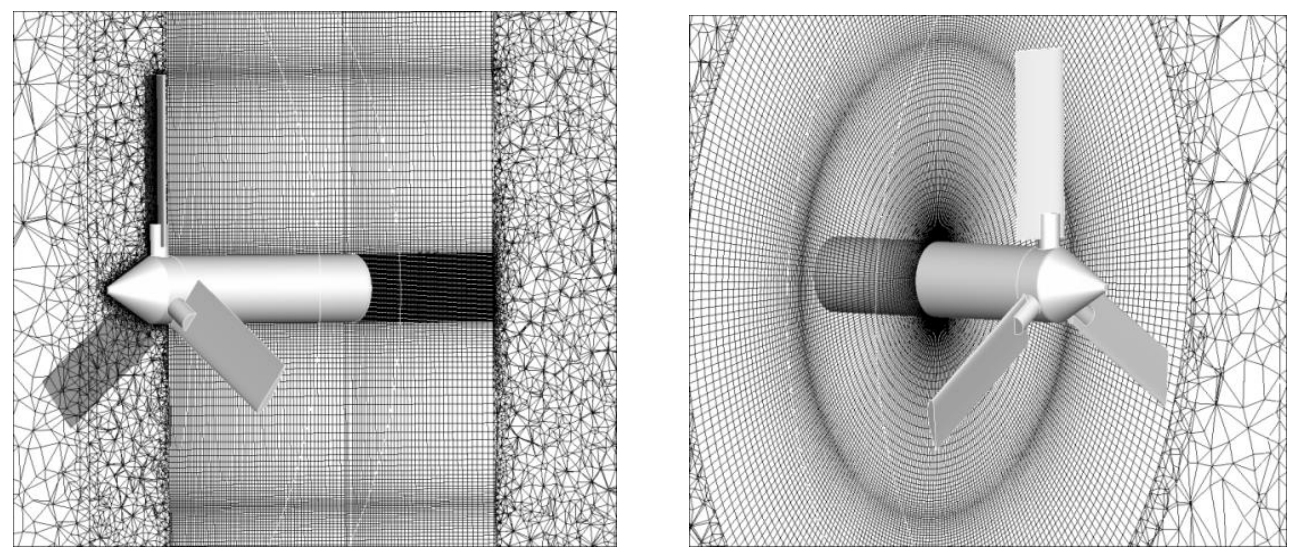

Fig. 3. Mesh refinement behind the rotor.

state technique, it is a suitable method to determine how design parameters (such as tip speed ratio, wind velocity and pitch angle) affect the wake aerodynamic behavior as approved by Rodrigues and Lengsfeld (2019a,b). They established a computational frame work using the MRF method to identify parameters influencing the wake.

The grid was generated with the Gambit software and a suitable size functions to optimize the nodes distribution on the rotor blade surfaces. Over the rotor and for the outer region, we adopted the tetrahedral mesh technique to make a high quality of the generated grid and control the mesh volume development. While, for the innerregion, the hexahedral mesh was chosen to obtain more structured mesh refinement. Figure 3 shows the grid refinement in the inner-region downstream of the wind turbine with respect to a node skewness value less than 0.761 .

The wall $y^{+}$value is given by the Eq. (1) as following:

$$
y^{+}=\frac{y_{p} u_{t}}{v}
$$

Where $y_{p}$ is the first grid point distance from the rotor, $u_{t}$ is the friction velocity, and $v$ is the kinematic viscosity.

The $y^{+}$value for the first elements from the rotor wall was around 30 . The non-constant value of the $y^{+}$is due to the rotation speed of the rotor and the elements position on the rotor as mentioned by Tian et al. (2015). For that, we utilized the Standard wall functions for modeling the turbulent effects below the first grid element to increase the solution accuracy.

\subsection{Mathematical Model and Solver Setting}

The tip and root vortices detached from each blade are the origins of the helical aspect of the wind turbine wake and the meandering phenomenon occurring during operation (Sherry et al. 2013b). The accuracy of a three-dimensional simulation depends on the choice of the turbulence model, which can carry out the helical behavior of the wake and the best prediction of the pressure coefficient along each blade section. For that, different turbulence models were investigated to study the wind turbine performances. Vucina et al. (2016) performed numerical investigations to optimize the shape of HAWT blades. During simulations, the flow was assumed to be fully turbulent at high Reynolds number equal to $10^{6}$, and they concluded 
that the $k \omega$-SST turbulence model gives the best results compared to the experiments in these conditions. AbdelSalam and Ramalingam (2014) conducted a steady-state three-dimensional simulation using the Fluent software to model a HAWT with high fidelity to the rotor geometry. The implemented Standard $k-\varepsilon$ turbulence model, have proved its ability versus experimental results and actuator disc approach using the modified $k-\varepsilon$ model by El Kasmi and Masson (2008). Derakhshan and Tavaziani (2015), and Lanzafame et al. (2013), also affirmed that the Standard $k-\varepsilon$ model gives a better accuracy with the experimental results for wind speed between 5 to $10 \mathrm{~m} / \mathrm{s}$ and low Reynolds number at the rotor blades. In this work, the Standard $k-\varepsilon$ turbulence model was used for its advantages cited previously for fully geometry approach. The turbulence level used in the computations was set equal to 0.1 with a hydraulic diameter equal to $0.888 \mathrm{~m}$ in respect with the CRTEn wind tunnel geometrical characteristics. The transient condition was activated to well model the unsteady effects, the blade interactions, and the wake generation. The solver was set as pressurebased with absolute velocity formulation. The Moving Reference Frame (MRF) boundary condition was activated for the rotating zone fixing the rotational speed.

The fixed reference frame was modeled using the governing equations as follow :

+ The continuity equation:

$\frac{d \rho}{d t}+\nabla \cdot(\rho \vec{v})=0$

Where, $\rho$ is the density of the fluid and $\vec{v}$ is the velocity vector.

+ The momentum equation:

$$
\frac{d}{d t}(\rho \vec{v})+\nabla \cdot(\rho \vec{v})=-\nabla p+\nabla \bar{\tau}+\rho \vec{g}+\vec{F}
$$

Where $p$ is the pressure, $\rho \vec{g}$ and $\vec{F}$ are the force of gravitation and the external forces respectively and finally the stress tensor $\underset{\tau}{\bar{\tau}}$ given as follow:

$\bar{\tau}=\mu\left[\left(\nabla \vec{v}+\nabla \vec{v}^{T}\right)-\frac{2}{3} \nabla \cdot \vec{v} I\right]$

Where $\mu$ is the molecular viscosity and $I$ is the tensor unit.

When the flow turbulence is cinsidered, the equations of conservation are time-averaged and then the Reynolds-averaged Navier-Stokes (RANS) equations are obtained. The equations can be written in a Cartesian tensor form as:

$$
\begin{aligned}
& \frac{d \rho}{d t}+\frac{d}{d x_{i}}\left(\rho u_{i}\right)=0 \\
& \frac{d}{d t}\left(\rho u_{i}\right)+\frac{d}{d x_{i}}\left(\rho u_{i} u_{j}\right)=-\frac{d \rho}{d x_{i}}+\frac{d}{d x_{j}}\left[\mu\left(\frac{d u_{i}}{d x_{i}}+\frac{d u_{j}}{d x_{j}}-\frac{2}{3} \delta_{i j} \frac{d u_{i}}{d x_{j}}\right)\right]+\frac{d}{d x_{i}}\left(-\rho \overline{u_{i}^{\prime} u_{j}^{\prime}}\right)
\end{aligned}
$$

Here $u_{i}$ and $u_{i}{ }^{\prime}$ represent the instantaneous and fluctuating velocity terms.
Given the fluid velocity $u_{i}$ as a function of position and time, the average fluid velocity is written as:

$u_{i}=\overline{u_{i}}+u_{i}^{\prime}$

The standard $k-\varepsilon$ model is a semi-empirical formulation (Launder and Spalding 1972) based on transport equations for the turbulence kinetic energy $k$ and its dissipation rate $\varepsilon$. In the derivation of the $k-\varepsilon$ model, the flow was assumed fully turbulent, and the effects of molecular viscosity are negligible. The standard $k-\varepsilon$ is, therefore, valid only for fully turbulent flows.

The turbulence kinetic energy, $k$ and its rate of dissipation $\varepsilon$ are obtained from the following transport equations:

$$
\begin{aligned}
& \frac{d}{d t}(\rho k)+\frac{d}{d x_{i}}\left(\rho k u_{i}\right)=\frac{d}{d x_{j}}\left[\left(\mu+\frac{\mu_{t}}{\sigma_{k}}\right) \frac{d k}{d x_{j}}\right]+G_{k}+G_{b}-\rho \varepsilon-Y_{M}+S_{K} \\
& \frac{d}{d t}(\rho \varepsilon)+\frac{d}{d x_{i}}\left(\rho \varepsilon u_{i}\right)=\frac{d}{d x_{j}}\left[\left(\mu+\frac{\mu_{t}}{\sigma_{\varepsilon}}\right) \frac{d \varepsilon}{d x_{j}}\right]+C_{1 \varepsilon} \frac{\varepsilon}{k}\left(G_{k}+C_{3 \varepsilon} G_{b}\right)-C_{2 \varepsilon} \frac{\varepsilon^{2}}{k}+S_{\varepsilon}
\end{aligned}
$$

Where $G_{k}$ is the turbulence kinetic energy due to mean velocity gradients, $G_{b}$ is the turbulence kinetic energy due to buoyancy, $Y_{M}$ is the contribution of the fluctuating dilatation in compressible turbulence to overall dissipation rate, $C_{1 \varepsilon}, C_{2 \varepsilon}$, and $C_{3 \varepsilon}$ are experimentally determined constants, $\sigma_{\varepsilon}$, and $\sigma_{k}$ are turbulent Prandtl numbers determined experimentally for $k$ and $\varepsilon, S_{k}$ and $S_{\varepsilon}$ are the source term.

The turbulent viscosity is computed as:

$\mu_{t}=\rho C_{\mu} \frac{k^{2}}{\varepsilon}$

Where, $C_{\mu}$ is a constant equal to $0.09, C_{1 \varepsilon}=1.44$, $C_{2 \varepsilon}=1.12$, TKE Prandlt number $=1$, and TDR Prandlt number $=1.3$.

The SIMPLE solver and the Second Order Upwind Scheme were used for the spatial discretization of the density, the momentum, and the turbulent viscosity equations. The Second Order Implicit Formulation was used, providing better results than reducing interpolation errors and false numerical diffusion. The under-Relaxation Factor was adjusted to facilitate the convergence. Two control monitors of the iterative solution were used to ensure the convergence. The simulation process was considered to be converged when the residuals decrease under the value of $10^{-6}$ for the velocity components of the momentum equation and $10^{-4}$ for the continuity equation, the turbulence kinetic energy $k$ and the dissipation rate $\varepsilon$.

The time-dependence and the grid-dependence studies was performed. In fact, the choice of the time step influences is an essential step in the numerical iterative process. For that, three different time steps was tested $10^{-2}, 10^{-3}$ and $10^{-4}$ for the momentum coefficient, as shown in Fig. 4. The results show that the $\Delta t=10^{-3}$ was the appropriate time step to be used during the simulations. 


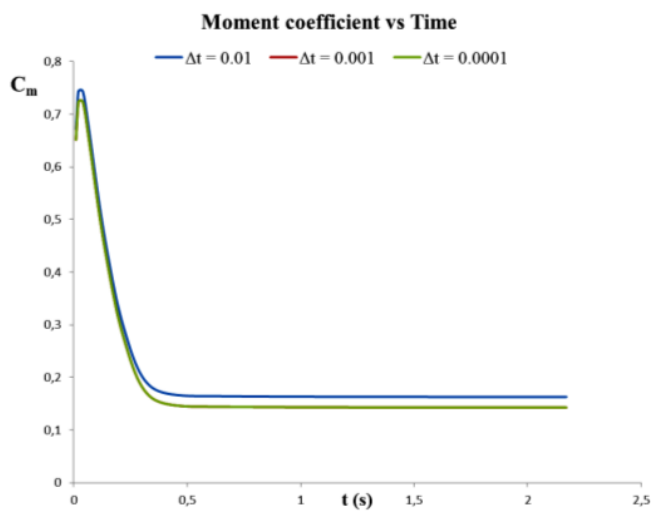

Fig. 4. Independence results of the computed moment coefficient of the rotor with time for three different time steps $10^{-2}, 10^{-3}$ and $10^{-4}$.

The grid-dependence study was, also, performed for three different mesh numbers of 2855018,3101 569, and 3324776 cells referred as mesh 1, mesh 2 and mesh 3 respectively. The velocity profiles in the near wake of the HAWT model at three different position $x / d=0.15, x / d=0.4$ and $x / d=1.33$ was illustrated respectively in Fig. 5.

Results show that good agreement in the local values of velocity is observed between mesh 2 and 3 . Since the variation in velocity between these two mesh densities was negligible, it was decided to proceed with the mesh settings and parameters used for mesh 3 to obtain results with more accuracy.

\section{RESUltS AND DiscuSSIONS}

\subsection{Flow Characteristics over a Rotating Blade}

The above simulations permitted us to illustrate the three-dimensional axial velocity behind the rotor, as shown in Fig. 6. The detached tip and root vortices convected in the wake as rigid bodies, giving a vortex tubes with a helical geometry during their displacement. The generated wake behind the wind turbine rotor, as well as the strength of the tip vortices, are mainly dependent on the flow separation over the rotor blades.

As shown in Fig. 7-a), a separation zone is established on the suction side under the effects of the non-symmetry shape of the airfoil, the pitch angle, and the centrifugal force. As a result, the centrifugal force convect the fluid in a radial direction as illustrated in Fig. 7-b).

\subsection{Validation of the Obtained Results}

In this section, the numerical results will be validated using the experimental results obtained in the CRTEn wind tunnel by Dahmouni et al. (2017). They conducted an experimental study to analyze the meandering phenomenon of tip vortices in the near wake of a three-bladed HAWT model for different tip speed ratios $\lambda=\left[\begin{array}{ll}3.50 & -5.22\end{array}\right]$. The model was tested in the wind tunnel section for a blockage ratio of $13.88 \%$ which is suitable for the considered range of tip speed ratio.

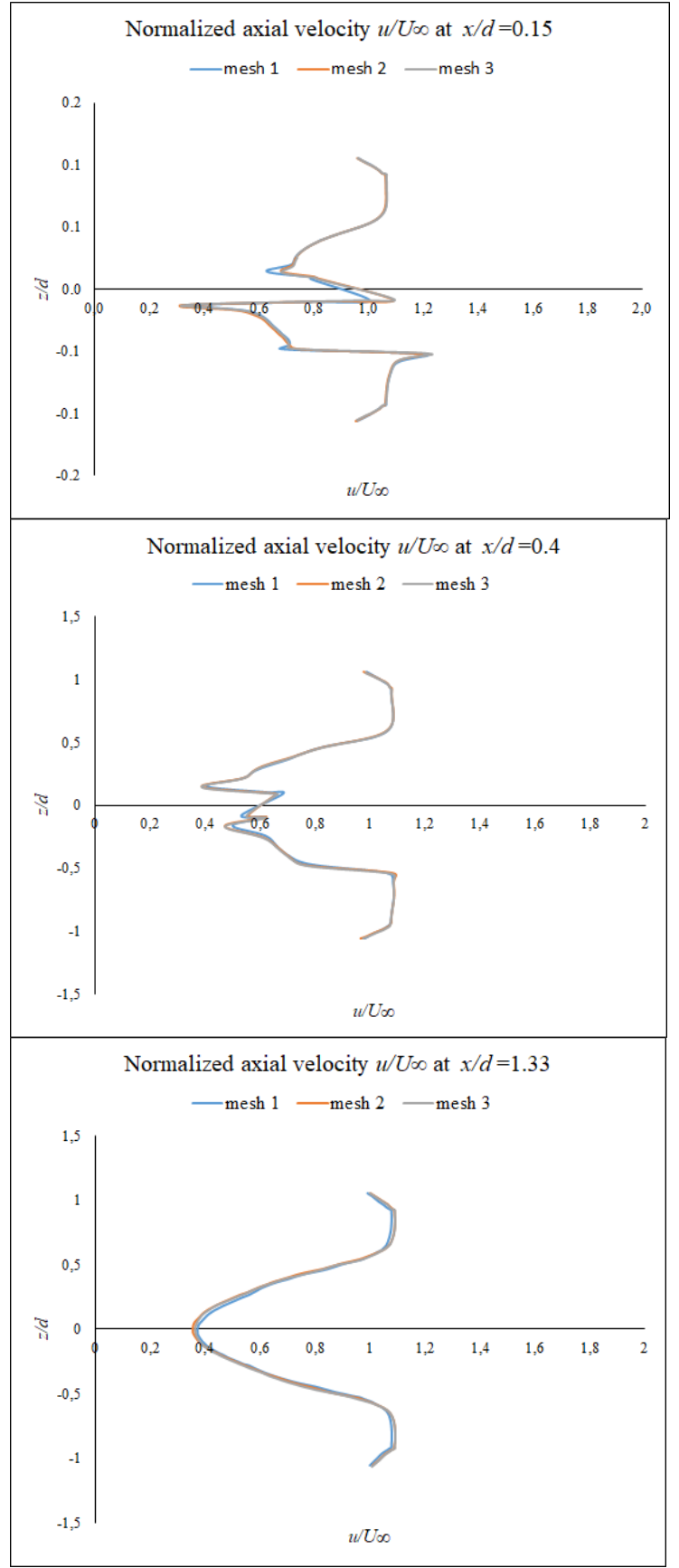

Fig. 5 Velocity profiles at different positions behind the rotor for different grid number at $x / d$ $=0.15, x / d=0.4$ and $x / d=1.33$ respectively

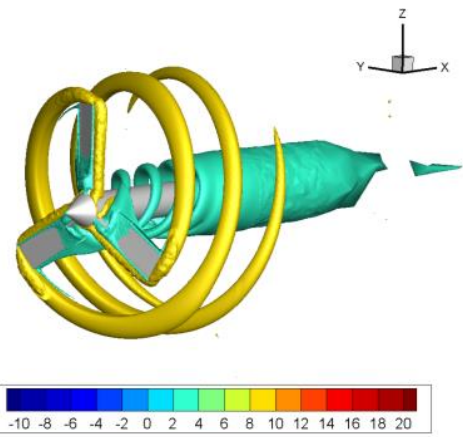

Fig. 6. Three dimensional axial velocity isosurfaces behind the wind turbine model. 


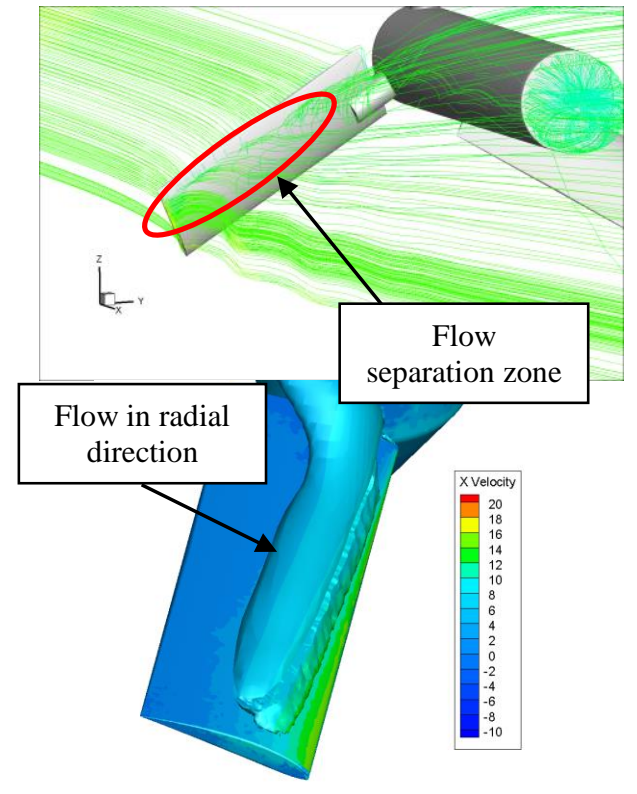

Fig. 7. a) The three dimensional streamlines behind the rotor blade and $b$ ) the axial velocity in the radial direction.

The non-intrusive laser optical PIV technique, was used for instantaneous bi-dimensional velocity field measurements. It consists of: a dual-pulsed $\mathrm{Nd}$ :YAG laser with $65 \mathrm{~mJ}$ energy output, a FlowSense CCD camera with resolution up to 1600x1200 pixels, a synchronizer acting as an external trigger, a high volume oil droplet-seeding generator and an optical sensor for rotational speed measurement. Processing of the raw images was carried out through the Dantec Dynamic Studio $\mathrm{v} 2.30$.

The wind speed ratio used for the validation was equal to 4.08 , corresponding to $8 \mathrm{~m} / \mathrm{s}$ of wind velocity upstream to the rotor to reproduce the same experimental conditions. The generated wake downstream of the rotor was explored for the axial velocity component $\quad$ in the $(X, Y)$ plane. Figure 8 illustrates the normalized two-dimensional axial velocity obtained by the CFD calculations and during experiments. The results show a qualitative good agreement with the experimental results. We can observe that the tip and root vortices detached from each blade in the near wake of the rotor are clearly apparent. This coherence can be approved by comparing the numeric axial velocity at the center of each tip vortex with the obtained experimental results, as shown in Fig.9.

The computed results show a good agreement with the experimental results. The slight observed differences and the shift between the positions of the tip vortices centers were a result of two principal reasons. The first one was the limit of mesh grid number related to the station computing capacities and the second reason was the meandering phenomenon of the tip vortices which is defined as an instantaneous fluctuation of the vortex core position (Devenport et al. 1996; Roy et al. 2011). In wind turbine case, as mentioned by (Bandyopadhyay et al. 1991; Sherry et al. 2013a,b and Dahmouni et al. 2017), the meandering phenomenon is due to the instantaneous changes in the vortex strength, the helical nature of the vortex tubes and the interaction between instabilities in the wake. However, no offset was observed for the first tip vortex because the meandering phenomenon increases as the vortices are far away from the rotor where the interactions between the tip vortices and the wake becomes more critical.

\subsection{Classification of Kelvin Wave's}

In stability analysis, three parameters describe the Kelvin waves which are the axial wavenumber $l$, the azimuthal wavenumber $m$, and the complex frequency $\omega$. The characteristics of a small perturbation in the form of eigenmodes can be defined as follows: $\left(u_{r}^{\prime}, u^{\prime} \theta, u^{\prime} z, p^{\prime}\right)=[u(r), v(r), w(r), p(r)] \exp (\mathrm{i} l z+\mathrm{i} m \theta-$
$\mathrm{i} \omega \mathrm{t})+\mathrm{cte}$

According to their azimuthal wavenumber $m$, the propagating Kelvin waves along the vortex tube were classified into three principal families, as described by Fabre et al. (2006), known as the axisymmetric mode for $m=0$ (Fig. 10-a), the helical mode for $m= \pm 1$ (Fig. 10-b) and the multi helices mode corresponding to wavenumbers (Fig. 10-c).

Each of the cited modes has specific physical characteristics. The axisymmetric mode $(m=0)$, creates an axial-flow inside the vortex which causes core deformation by a stretching-compressing mechanism.

However, the helical and multi-helical modes present more complex structures and interaction phenomenon than the axisymmetric waves. As classified by Saffman (1992), Fabre et al. (2006) and Thomas (2009), four different families of waves are distinguished for the helical mode $(m= \pm 1)$ : the displacement waves, the core waves, the purely viscous waves, and the spiral waves noted as $D, C, V$ and $L$ branches respectively. In the case of double-helical mode $(m=2)$, only three family branches are identified by similarity with the helical mode noted as following $C, V$ and $L$ branches. In particular, the $L$ family was decomposed into two sub-families called $L 1$ and $L 2$. The Figures 11, 12 and 13 illustrate an example of the families for $m=1, m=2$ and the $L 1$ branches of $m=2$ respectively.

The major difference between the double helix subfamilies is the location of the vorticity quadrupole which is totally situated within the vortex core in the case of $\mathrm{C}$ and $\mathrm{V}$ branches presented in Fig. 12-a) and Fig. 12-b) and outgoing the vortex core in other cases as presented in Fig. 12-c), Fig.13-a) and Fig.13-b). 
M. M. Oueslati et al. / JAFM, Vol. 13, No. 5, pp. 1421-1435, 2020.
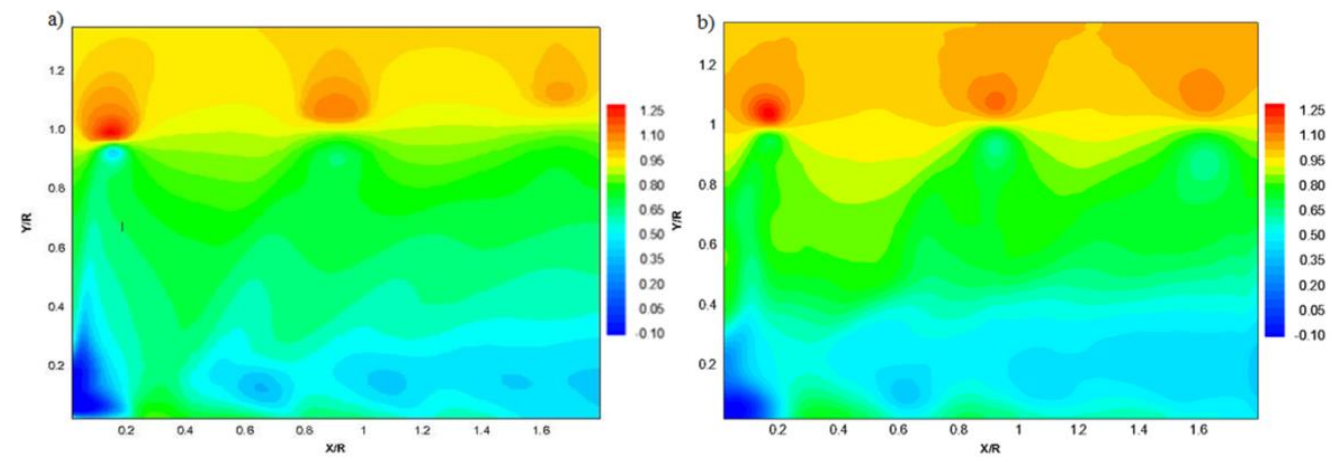

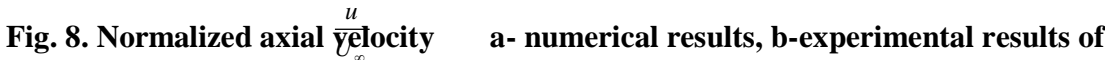
Dahmouni et al. (2017).

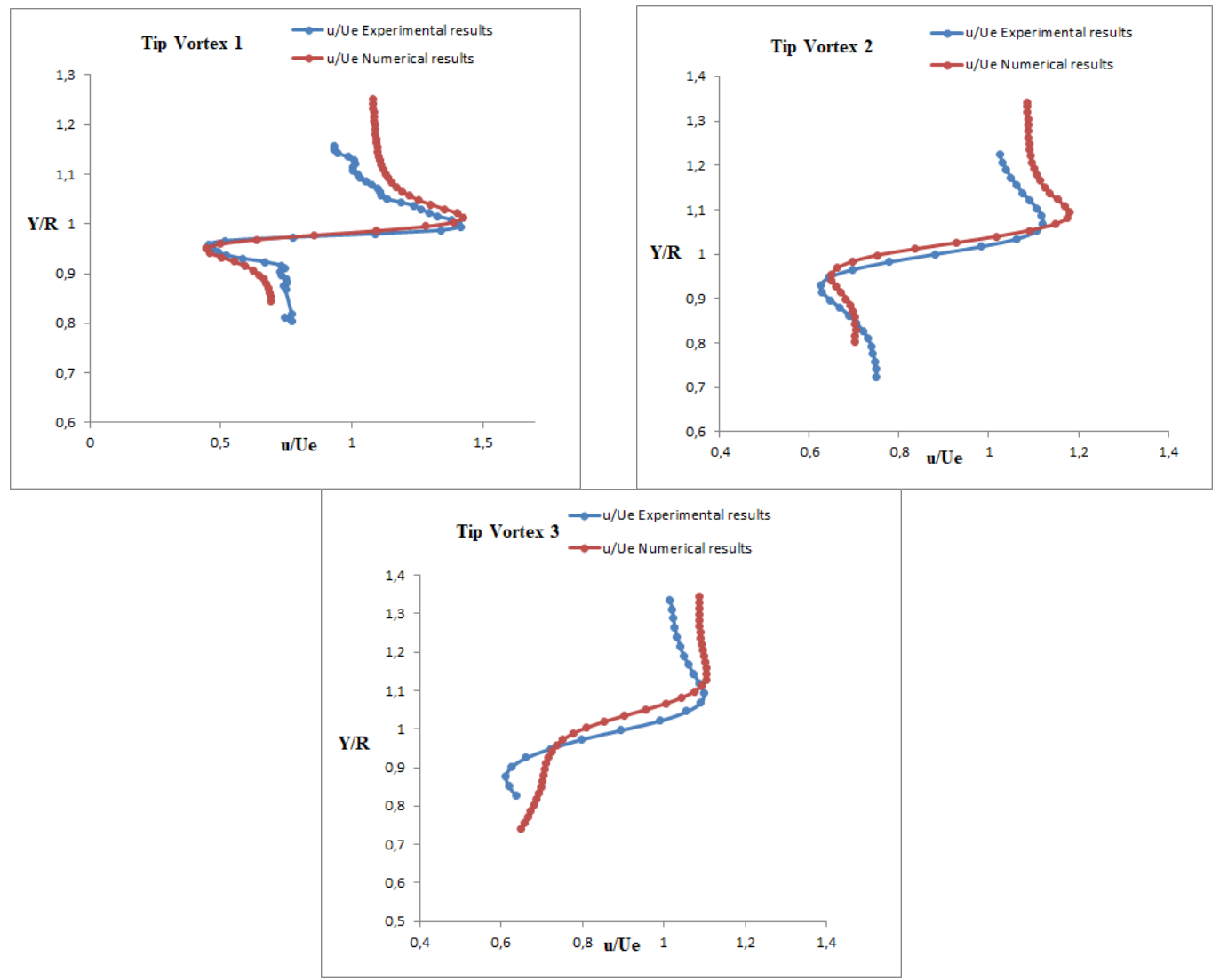

Fig. 9. Numerical axial velocities at the center of each tip vortices compared to the experimental results obtained by Dahmouni et al. (2017).
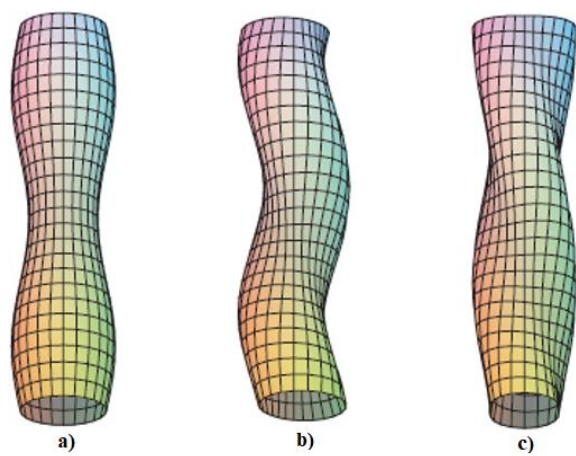

Fig. 10. Axisymmetric mode, the helical mode and the multi helices mode corresponding to $m=0, m= \pm 1$ and $|m|>1$ respectively (Fabre et al. 2006). 

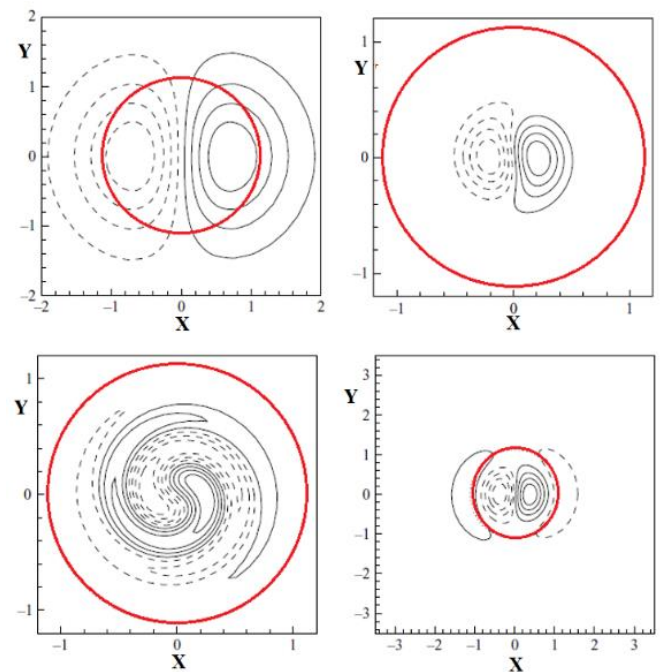

Fig. 11. Families of the helical mode $(m=1) \mathrm{D}, C$, $V$ and $L$ respectively (Fabre et al. 2006).

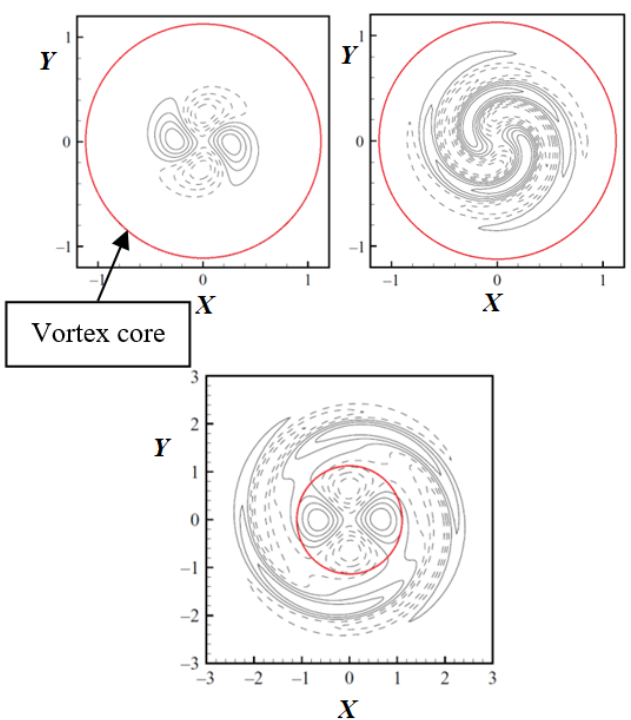

Fig. 12. Double-helix mode families $C, V$, and $L$ for wave number $m=2$ (Fabre et al. 2006).

\subsection{Analysis of Velocity Gradient Tensor Components According to Kelvin Theory}

As detailed previously, the flow separation features involve a high perturbation affecting the wake structure downstream of the rotor, its expansion, its intensity and the meandering phenomenon. The tip and root vortices are the most energetic elements in the wake, their strength depends mainly on the type of deformations acting on it. For that, it's necessary to analyze those phenomena which generate an extra aerodynamic and mechanical loads on the rotor. To study the tip vortex tube deformation in the HAWT near wake, we will focus our analysis on the concordance between the Kelvin wave modes and the velocity gradient tensor components which gives a clear idea on the different physical phenomena occurring during the development of the helical tip vortex.
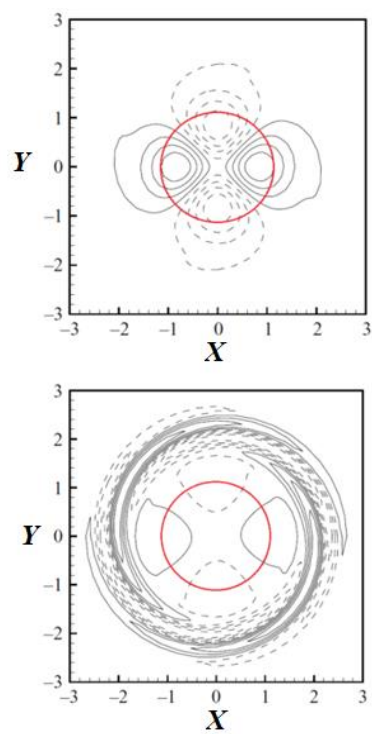

Fig. 13. The $L 1$ sub-branch family of $L$ family branch of Double-helix mode for different frequencies (Fabre et al. 2006).

For incompressible flow, the three-dimensional form of the velocity gradient tensor is given as follow:

$$
\nabla u=\left(\begin{array}{lll}
\frac{\partial u}{\partial x} & \frac{\partial v}{\partial x} & \frac{\partial w}{\partial x} \\
\frac{\partial u}{\partial y} & \frac{\partial v}{\partial y} & \frac{\partial w}{\partial y} \\
\frac{\partial u}{\partial z} & \frac{\partial v}{\partial z} & \frac{\partial w}{\partial z}
\end{array}\right)
$$

Figure 14 shows the velocity gradient tensor components for the first wind turbine tip vortex.

Generally, the velocity gradient tensor is decomposed into a symmetric tensor called the rate of deformation tensor and a skew-symmetric tensor which represents the rotation rate known as the vorticity tensor.

Each tensor component indicates an information about the type of deformation acting on the tip vortex. So, it will be compared against the Kelvin wave modes to pick up the different mechanisms occurring inside the tip vortex tubes.

The components $\frac{\partial u}{\partial x}, \frac{\partial u}{\partial y}$, and $\frac{\partial w}{\partial z}$, have the same variation features. They have a quadripole structure with a sign alternatively positive and negative along the diagonal directions. As described by Fabre et al. (2006), this can be explained by the wavering phenomenon in the vortex tube as provided by the Reynolds stress component $\overline{u^{\prime} v^{\prime}}$ in Fig. 15.

Besides, it is remarkable that there are similarities in the apparent features with the double-helix modes of Kelvin waves corresponding to a wavelength equal to $m=2$. 
M. M. Oueslati et al. / JAFM, Vol. 13, No. 5, pp. 1421-1435, 2020.
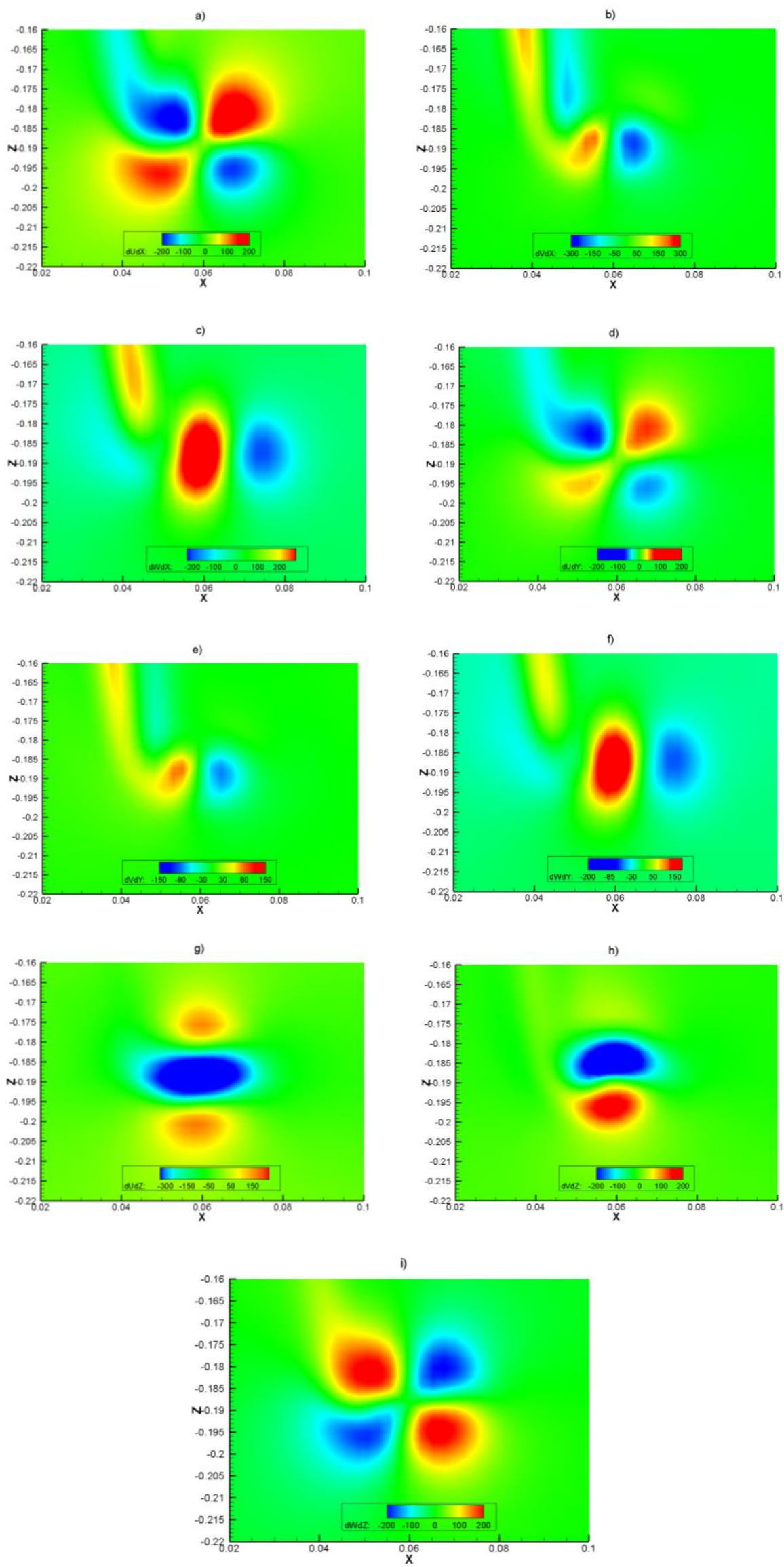

Fig. 14. Velocity gradient tensor components. 


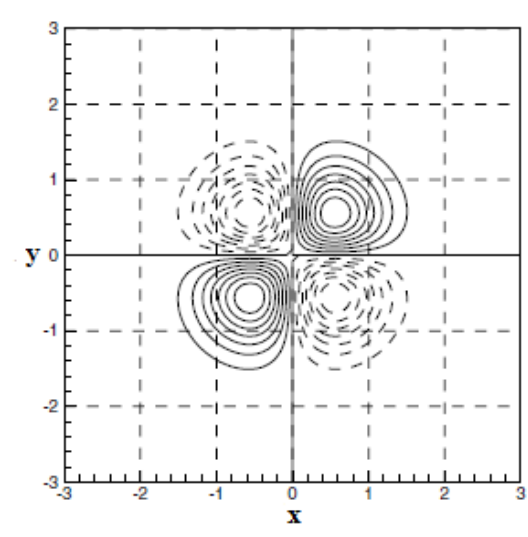

Fig. 15. Reynolds stress component $\overline{u^{\prime} v^{\prime}}$.

To find the family branch of the kelvin wave, we superposed the iso-values of $\partial u / \partial x, \partial u / \partial y$, and $\partial w / \partial z$ on the vorticity magnitude contours of the tip vortex, as presented in Fig. 16 and Fig. 17.

Figures show that the velocity gradient tensor components are not confined only into the core vortex but also extends to the outer parts like a Kelvin wave belong to the $L$ branch family and especially the $L 1$ branch as shown in Fig. 13. A low axial wavenumber and a substantial increase of the damping rate are observed for the $\partial u / \partial x$ and $^{\partial u / \partial y}$ components. However, the $\partial w / \partial z$ component is characterized by a higher axial wavenumber. The physical signification of such quadripole is an elliptical deformation of the vortex core by increasing the base-flow vorticity along one direction and decrease it in the perpendicular direction.

We can also identify, the helical mode of Kelvin waves corresponding

the azimuthal wavenumber equal to $m=1$. Figure 14 show that $\partial v / \partial x_{\text {and }} \partial v / \partial y$ presents a vorticity dipole aspect. By analyzing the branches of families in Fig. 11, we can confirm that they belong to the $D$ branch family. In this case, the Kelvin wave rotates in the opposite direction of the left-propagating core vortex for all wavelengths.

The velocity gradient components increase in the left part and decrease in the other part, so the helical displacement of the tip vortex go on the flow direction. However, the $\partial v / \partial z$ component illustrates a vertical dipole corresponding to the vertical displacement in $z$ direction. As a result, the observed tip vortex meandering phenomenon in the HAWT near wake correspond to a Kelvin waves of helical mode with azimuthal wave number equal to 1 belonging to the $D$ branch family.

On another hand, the velocity gradient components $\partial u / \partial z, \quad \partial w / \partial x$, and $\partial w / \partial y$ composed of three vortices in vertical or horizontal directions, respectively, corresponding to a secondorder azimuth symmetry. Figure 18 illustrates the vorticity magnitude contours superposed on the velocity gradient contours of each component. We remarked an elliptic stretching-compression perturbation exerting forces on the vortex in the $z$ direction for $\partial w / \partial x$ and $\partial w / \partial y$ components and the $x$-direction for $\partial u / \partial z$ component. Then, the propagated waves in the vortex tube have the characteristics of axisymmetric Kelvin waves corresponding to azimuth wavenumber equal to $m=0$. This type of instability applies an axial pressure forces in the $x$ and $z$ directions which deforms the tip vortex, changes its orientation angle and accelerates it.
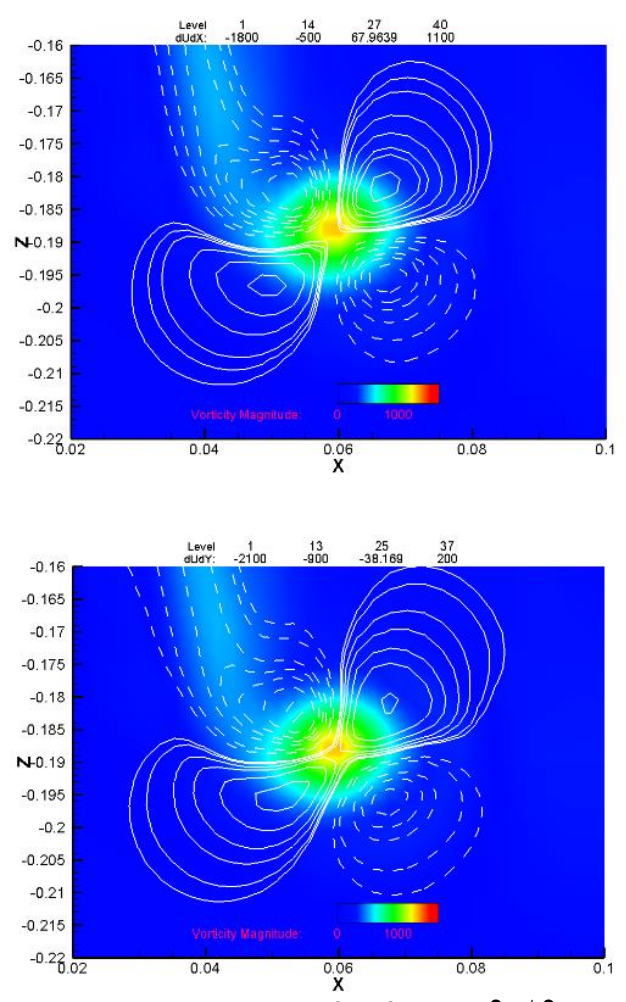

Fig. 16. Contours of $\partial u / \partial x$ and $\partial u / \partial y$ superposed on the vorticity magnitude respectively.

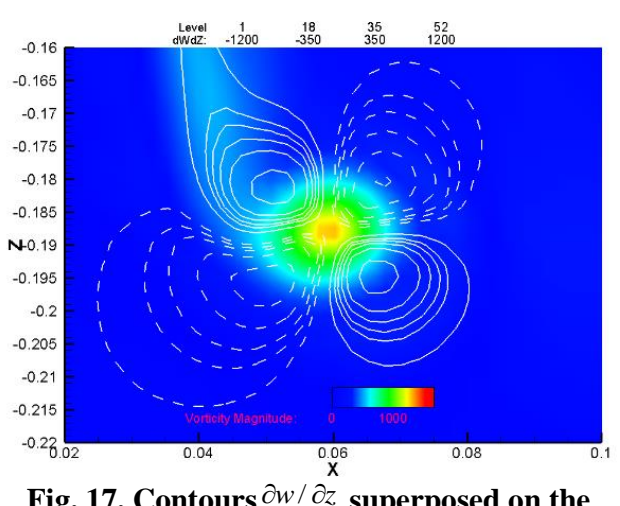

Fig. 17. Contours $\partial w / \partial z$ superposed on the vorticity magnitude map.

Furthermore, the vertical and lateral dipoles structures observed in each component are responsible for increasing and decreasing the vorticity magnitude.

This phenomenon correspond to the case of a 
double-helix Kelvin wave for an azimuth wavenumber equal to $m=2$ belonging to the $L 1$ family branch.

In conclusion, the $\partial u / \partial z, \partial w / \partial x$, and $\partial w / \partial y$ components illustrate the propagation of axisymmetric and double-helix Kelvin waves corresponding to $m=0$ and $m=2$ in combined form.
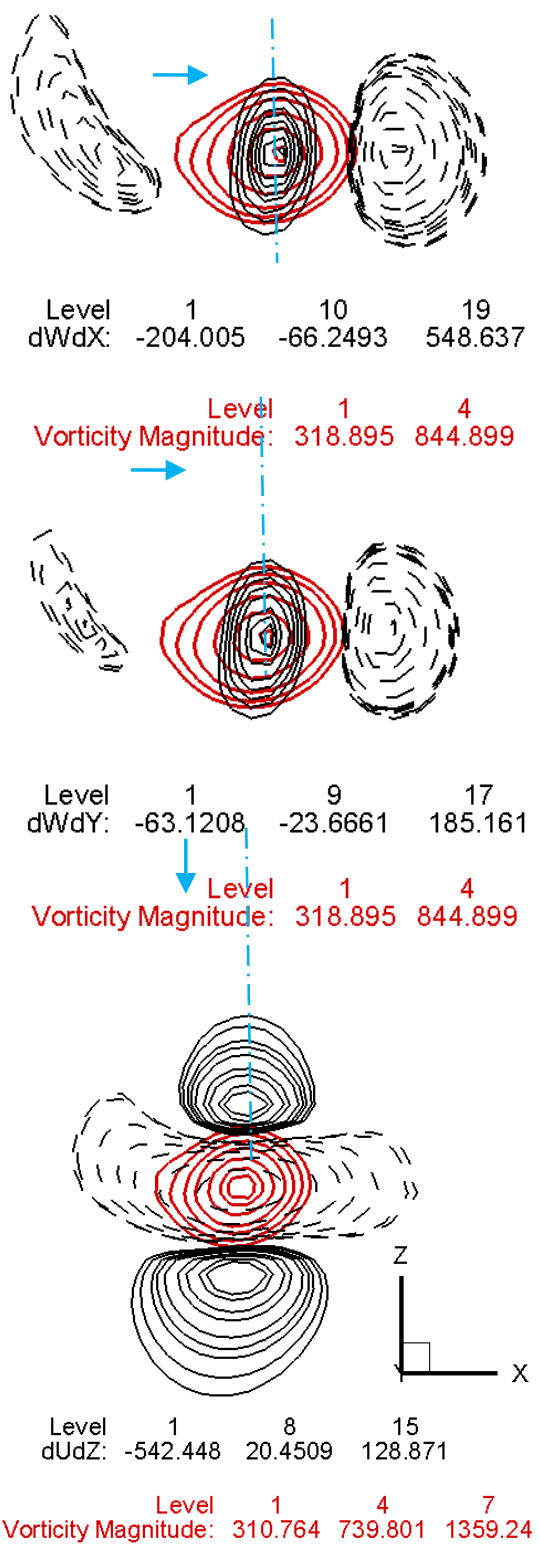

$\begin{array}{ccc}\text { Vorticity Magnitude: } 310.764 & 739.801 & 1359.24\end{array}$

Fig. 18. The $\partial u / \partial z, \partial w / \partial x$, and $\partial w / \partial y$ components of the velocity gradient tensor.

In addition to the stretching-compression phenomenon that undergoes the tip vortex, the present instabilities change also the vortex orientation during the wake expansion. In more detail, Fig. 19 illustrates the $\partial u / \partial z$ at three different $(x, z)$ planes respectively at $30^{\circ}, 50^{\circ}$, and $70^{\circ}$.

Figure 20 represents the tip vortex in the $30^{\circ}, 50^{\circ}$, and $70^{\circ}$ planes, respectively. The orientation angle of the tip vortex in the plane $30^{\circ}$ was equal to $\beta=$ $10^{\circ}$. As the distance between the tip vortex and the blade growth the vortex inclination angle increases. In our cases, it is equal to $\beta=22^{\circ}$ and $\beta=29^{\circ}$ corresponding respectively to the planes $50^{\circ}$ and $70^{\circ}$.

As a result, the vortex tube undergoes a twist, its core radius increase and an elliptical deformation affects its shape during the wake development as the distance increase behind the HAWT rotor.

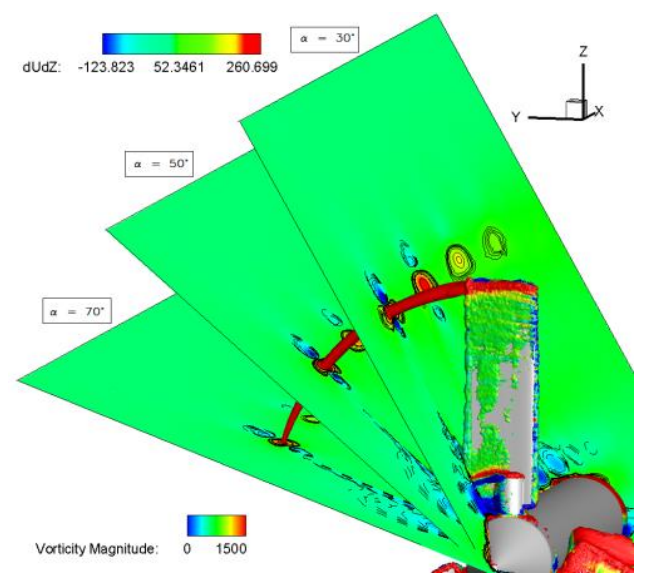

Fig. 19 The $\partial u / \partial z$ at three different planes $30^{\circ}$, $50^{\circ}$ and $70^{\circ}$ respectively.
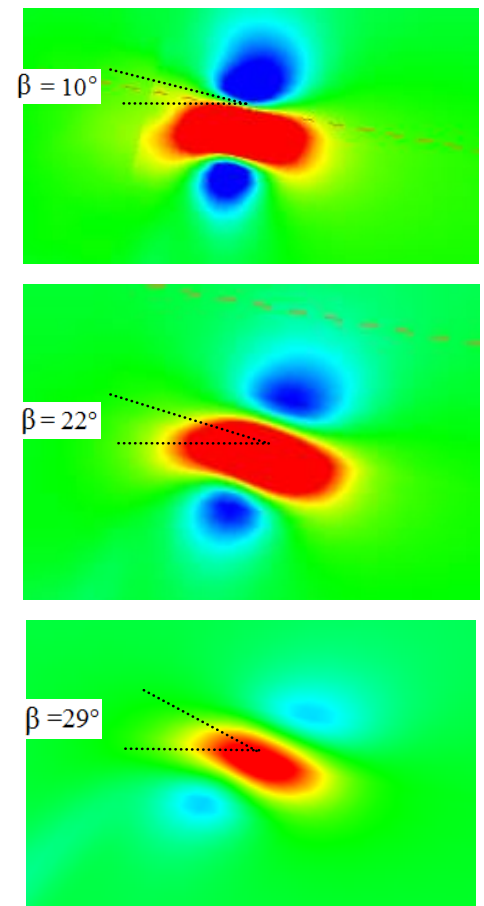

Fig. 20. Variation of the inclination angle of the tip vortex for each plane.

\section{CONCLUSION}

In this paper, three-dimensional simulations of a HAWT model was investigated using the Fluent 6.3.26 software to analysis the tip vortices 
deformation. The obtained wind turbine near wake was validated with experimental results processed in the CRTEn wind tunnel. The identification of the Kelvin wave's modes was detailed, allowing us to identify the various instabilities and deformations affecting the helical tip vortices in the near wake of a HAWT.

By superposing the velocity gradient tensor components on the vorticity magnitude, we have approved the presence of the wavering phenomenon into the vortex tube. Based on the Kelvin wave's analysis, three different modes were branded as:

- $\quad$ The helical mode corresponding to $m=1$ belonging to the $D$ family branch, has an essential role in the establishment of the meandering phenomenon of the tip vortex.

- The double-helix wave mode corresponding to wavelength equal to $m=2$ belonging to the $L 1$ family branch, is responsible for the elliptical deformtion of the vortex core by increasing the baseflow vorticity along one direction and decrease it in the perpendicular direction.

- The combined mode: axisymmetric and double-helix modes. This mixed-mode has a significant effect on the tip vortices shape. It applies axial pressure forces on the tip vortex in the $x$ and $z$ directions as well as the stretching-compression phenomenon.

Future investigations will focus on the analysis of the instability's effects on the far wake behavior. The influence of Reynolds number as well as the geometric blade parameters on their evolution in the helical vortex tube will be also considered.

\section{In Memory of Professor Sassi Ben NASRALLAH}

We would like to pay tribute to our dear Professor Sassi BEN NASRALLAH, co-author of this paper, who left us suddenly on June 2017. Professor Sassi BEN NASRALLAH was for us an excellent teacher, a serious person and a scientific luminary. We lost more than just a colleague, but a scientific father.

\section{REFERENCES}

AbdelSalam, A. M. and V. Ramalingam (2014). Wake prediction of horizontal-axis wind turbine using full-rotor modeling. Journal of Wind Engineering and Industrial Aerodynamics 124, 7-19.

Arendt, S., D. C. Fritts and Ø. Andreassen (1998). Kelvin twist waves in the transition to turbulence. European Journal of MechanicsB/Fluids 17(4), 595-604.

Aseyev, A. S. and R. B. Cal (2016). Vortex identification in the wake of a model wind turbine array. Journal of Turbulence 17(4), 357-378.

Bandyopadhyay, P. R., R. L. Ash and D. J. Stead (1991). Organized nature of a turbulent trailing vortex. AIAA Journal 29(10), 1627-1633.

Bastine, D., B. Witha, M. Wachter and J. Peinke (2014). POD analysis of a wind turbine wakein a turbulent atmospheric boundary layer. In Journal of physics: conference series 524, 012153.

Bouffard, D. and U. Lemmin (2013). Kelvin waves in Lake Geneva. Journal of Great Lakes Research 39 (4), 637-645.

Chong, M. S., A. E. Perry and B. J. Cantwell (1990). A general classification of three- dimensional flow fields. Physics of Fluids A: Fluid Dynamics 2(5), 765-777.

Dahmouni, A. W., M. M. Oueslati and S. B. Nasrallah (2017). Experimental Investigation of Tip Vortex Meandering in the Near Wake of a Horizontal-Axis Wind Turbine. Journal of Applied Fluid Mechanics 10(6), 1679-1688.

Derakhshan, S. and A. Tavaziani (2015). Study of wind turbine aerodynamic performance using numerical methods. Journal of Clean Energy Technologies 3(2), 83-90.

Devenport, W. J., M. C. Rife, S. I. Liapis and G. J. Follin (1996). The structure and development of a wing-tip vortex. Journal of Fluid Mechanics 312, 67-106.

El Kasmi, A. and C. Masson (2008). An extended $\mathrm{k}-\varepsilon$ model for turbulent flow through horizontal-axis wind turbines. Journal of Wind Engineering and Industrial Aerodynamics 96(1), 103-122.

Fabre, D., D. Sipp and L. Jacquin (2006). Kelvin waves and the singular modes of the LambOseen vortex. Journal of Fluid Mechanics 551, 235-274.

Gebhardt, C. G., S. Preidikman and J. C. Massa (2010). Numerical simulations of the aerodynamic behavior of large horizontal-axis wind turbines. International Journal of Hydrogen Energy 35 (11), 6005-6011.

Grant, I., M. Mo, X. Pan, P. Parkin, J. Powell, H. Reinecke, K. Shuang, F. Coton and D. Lee (2000). An experimental and numerical study of the vortex filaments in the wake of an operational, horizontal-axis, wind turbine. Journal of Wind Engineering and Industrial Aerodynamics 85(2), 177-189.

Hunt, J. C., A. A. Wray and P. Moin (1988). Eddies, streams, and convergence zones in turbulent flows. Center for Turbulence Research Proceedings of the Summer Program, 193.

Jacquin, L., D. Fabre, D. Sipp, V. Theofilis and H. Vollmers (2003). Instability and unsteadiness of aircraft wake vortices. Aerospace Science 
and Technology 7(8), 577-593.

Jeong, J. and F. Hussain (1995). On the identification of a vortex. Journal of Fluid Mechanics 285, 69-94.

Kelvin, L. (1867). On vortex atoms. Proceedings of the Royal Society of Edinburg 6, 94-105.

Kelvin, L. (1880). Vibrations of a columnar vortex. Philosophical Magazine 10, 155-168.

Kleusberg, E., S. Benard and D. S. Henningson (2019). Tip- vortex breakdown of wind turbines subject to shear. Wing Energy 22(12), 1789-1799.

Lanzafame, R., S. Mauro and M. Messina (2013). Wind turbine CFD modeling using a correlation-based transitional model. Renewable Energy 52, 31-39.

Lanzafame, R., S. Mauro and M. Messina (2016). Numerical and experimental analysis of micro HAWTs designed for wind tunnel applications. International Journal of Energy and Environmental Engineering 7(2), 199-210.

Larsen, G. C., H. Madsen Aagaard, F. Bingöl, J. Mann, S. Ott and J. N. Sørensen (2007). Dynamic wake meandering modeling. Ris $\phi$ National Laboratory for Sustainable Energy, Technical University of Denmark. Report number: Risoe-R: No. 1607(EN). ISSN 01062840 ISBN 978-87-550-3602-4.

Launder, B. E. and D. B. Spalding (1972). In Mathematical Models of turbulence, Academic Press, London, England.

Lumley, J. L. (1967). The structure of inhomogeneous turbulent flows. In A. M Iaglom and V. I Tatarski (Ed), Atmospheric Turbulence and Radio Wave Propagation, Nauka, Moscow, 221-227.

Massouh, F. and I. Dobrev (2014). Investigation of wind turbine flow and wake. Journal of Fluid Science and Technology 9(3), 1-9.

Mongiovi, M. S., D. Jou, M. Sciacca (2018). Nonequilibrium thermodynamics, heat transport and thermal waves in laminar and turbulent superfluid helium. Physics Reports 726, 1-71.

Moshfeghi, M., S. Shams and N. Hur (2017). Aerodynamic performance enhancement analysis of horizontal axis wind turbines using a passive flow control method via split blade. Journal of Wind Engineering and Industrial Aerodynamics 167, 148-159.

Noura, B., I. Dobrev, R. Dizene, F. Massouh and S. Khelladi (2012). Experimental study of yawed inflow around wind turbine rotor. Proceedings of the Institution of Mechanical Engineers, Part A: Journal of Power and Energy 226(5), 664-673.

Premaratne, P, W. Tian and H. Hu (2016). Analysis of wind turbine wake characteristics using proper orthogonal decomposition (POD) and triple decomposition. In $46^{\text {th }}$ AIAA Fluid Dynamics Conference, Washington, D.C., USA.

Rodrigues, R. V. and C. Lengsfeld (2019a), Development of a computational system to improve wind farm layout, Part I: Model validation and near wake analysis. Energies 12(5), 940.

Rodrigues, R. V. and C. Lengsfeld (2019b), Development of a Computational System to Improve Wind Farm Layout, Part II: wind turbine wake interaction. Energies 12(7), 1328.

Roy, C., T. Leweke, M. C. Thompson and K. Hourigan (2011). Experiments on the elliptic instability in vortex pairs with axial core flow. Journal of Fluid Mechanics 677, 383-416.

Saffman, P. (1992). Vortex Dynamics. Cambridge University Press, Cambridge, UK.

Sarmast S., R. Dadfar, R. F. Mikkelsen, P. Schlatter, S. Ivanell, J. N. Sørensen and D. S. Henningson (2014). Mutual inductance instability of the tip vortices behind a wind turbine. Journal of Fluid Mechanics 755, 705731.

Sherry, M., Sheridan J. and D. Lo Jacono (2013a). Characterisation of a horizontal axis wind turbine's tip and root vortices. Experiments in Fluids 54(3), 1417.

Sherry, M., A. Nemes, D. Lo Jacono, H. M. Blackburn and J. Sheridan (2013b). The interaction of helical tip and root vortices in a wind turbine wake. Physics of Fluids 25(11), 117102.

Thomas, O. (2009). Simulations numériques de perturbations d'un tourbillon de Lamb-Oseen : application à l'éclatement tourbillonnaire dans les sillages des avions de transport. $\mathrm{Ph}$. D. thesis, the Paris VI Univer-sity, Paris, France.

Tian, W., B. Song, J. Van Zwieten, P. Pyakurel (2015). Computational fluid dynamics prediction of a modified Savonius wind wurbine with novel blade shapes. Energies 8(8), 7915-7929.

Tossas, L. A. M. and S. Leonardi (2013). Wind Turbine Modeling for Computational Fluid Dynamics. In Technical Report No. NREL/SR5000-55054. National Renewable Energy Laboratory.

Tran, T. and D. H. Kim (2015). The platform pitching motion of floating offshore wind turbine: A preliminary unsteady aerodynamic analysis. Journal of Wind Engineering and Industrial Aerodynamics 142, 65-81.

Tran, T., D. Kim and J. Song (2014). Computational fluid dynamic analysis of a floating offshore wind turbine experiencing platform pitching motion. Energies 7(8), 50115026. 
M. M. Oueslati et al. / JAFM, Vol. 13, No. 5, pp. 1421-1435, 2020.

Vermeer, L. J., J. N. Sørensen and A. Crespo (2003). Wind turbine wake aerodynamics. Progress in Aerospace Sciences 39(6), 467510.

Vučina, D., I. Marinić-Kragić and Z. Milas (2016). Numerical models for robust shape optimization of wind turbine blades. Renewable Energy 87, 849-862.

Weber, J. E. H. and P. Ghaffari (2014). Mass transport in internal coastal Kelvin waves. European Journal of Mechanics-B/Fluids 47, 151-157. 